\title{
PROGRESSING WAVES IN AN INFINITE NONLINEAR STRING
}

\section{B. A. FLEISHMAN}

1. Introduction. Consider the nonlinear one-dimensional wave equation

$$
u_{t t}-u_{x x}=-\alpha u-\beta u^{3} .
$$

This equation governs the small transverse displacement of a uniform taut string constrained by distributed nonlinear "springs" exerting a force $-\alpha u-\beta u^{3}$. The spring constants $\alpha$ and $\beta$, also the variables $t, x$ and $u$, are real-valued. The string is assumed to be of infinite length.

We wish to determine the types of waves which propagate in this medium at constant speed, and their propagation speeds, for various values of $\alpha$ and $\beta$. The problem reduces to that of interpreting the solutions of the nonlinear ordinary differential equation (5); the properties of these solutions, and the method of obtaining them, are discussed in $\S 2$. We proceed, in $\S \S 3,4$ and 5 , to illustrate the noteworthy features of the wave solutions of (1) by considering particular combinations of $\alpha$ and $\beta$.

Waves which travel at speeds greater (less) than one will be called supercritical (subcritical). In $\$ 3$ it is shown that, for given positive $\alpha$ and $\beta$, all continuous wave solutions are of the form (13), where the amplitude $a$ and (supercritical) velocity $c$ may be chosen arbitrarily. In contrast, wave solutions of the simple wave equation

$$
u_{t t}-u_{x x}=0
$$

may have arbitrary shapes, but travel only with velocities \pm 1 . In $\S 4$ we note a duality principle between sub- and supercritical waves when the signs of $\alpha$ and $\beta$ are changed. Finally, in $\$ 5$, we see that when $\alpha \beta<0$, there exist both periodic and nonperiodic waves, of suband supercritical type. In this case, also, it is possible to have discontinuous waves which travel at unit speed.

In $[1]^{1}$ the author and F. A. Ficken have demonstrated the existence of time-periodic solutions, with period $p$, of a related equation with forcing and damping terms,

Presented to the Society, April 26, 1958; received by the editors June 23, 1958 and, in revised form, August 9, 1958.

1 Numbers in brackets refer to the list of references at the end of the paper. 


$$
u_{t t}-u_{x x}=-2 k u_{t}-\alpha u-\beta u^{3}+b(x, t),
$$

where $k>0, \alpha>0,|\beta|$ is small, and the "forcing function" $b(x, t)$ has the period $p$ in $t$. The results in [1] are supplemented somewhat by those of the present paper, in that the periodic waves discussed here are, ipso facto, time-periodic solutions of (1).

The approach to this problem was suggested by a paper of van der $\mathrm{Pol}[3]$ on another nonlinear wave equation. In preparing the present paper, the author benefited from several discussions with J. B. Keller.

2. Ordinary differential equation of a nonlinear mass-spring system. A wave traveling with velocity $c$ must be represented by a function of the form

$$
u(x, t)=\psi(x-c t)=\psi(s)
$$

where

$$
s=x-c t .
$$

Therefore, substituting $u=\psi(x-c t)$ in (1) we obtain

$$
\left(c^{2}-1\right) \frac{d^{2} \psi}{d s^{2}}=-\alpha \psi-\beta \psi^{3}
$$

Suppose $c^{2} \neq 1$. Dividing through by $c^{2}-1$, and denoting differentiation with respect to $s$ by primes, we have

$$
\psi^{\prime \prime}=-\bar{\alpha} \psi-\bar{\beta} \psi^{3},
$$

where the constants $\bar{\alpha}$ and $\bar{\beta}$ are defined by

$$
\bar{\alpha}=\frac{\alpha}{c^{2}-1}, \quad \bar{\beta}=\frac{\beta}{c^{2}-1} .
$$

We have, then, to examine the solutions $\psi(s)$ of the nonlinear ordinary differential equation (5) for various values of $\bar{\alpha}$ and $\bar{\beta}$. Except for a brief consideration of discontinuous waves in $\S 5$, we shall be interested only in bounded solutions of (5) with continuous second derivatives. Associated with each nonperiodic solution of (5), for fixed $\alpha, \beta$ and $c$, is a wave of nonperiodic form propagating with velocity $c$. Similarly, to periodic solutions of (5) will correspond waves with periodic shapes.

Equation (5) governs the free, undamped motion of a unit mass attached to a nonlinear spring, where $s$ is the time variable, $\psi(s)$ denotes the displacement of the mass from equilibrium at the instant $s$, and the spring exerts a force $-\bar{\alpha} \psi-\bar{\beta} \psi^{3}$. If $\bar{\alpha}>0$, the spring force 
is attractive for small displacements $\psi$, whatever the sign of $\bar{\beta}$. These cases have been treated in detail in studies of nonlinear vibrations, e.g., in the book by Stoker [4]. The cases with $\bar{\alpha}<0$ (repulsive force for small $|\psi|$ ) have not been studied as thoroughly for they are of less physical interest; but they can be treated by the same methods.

A first integral of (5) is obtained by multiplying through by $2 \psi^{\prime}$ and integrating, to give

$$
\psi^{\prime 2}+\bar{\alpha} \psi^{2}+\frac{\bar{\beta}}{2} \psi^{4}=h .
$$

Solving for $\psi^{\prime}=d \psi / d s$, separating variables and integrating, we obtain $s$ as a function of $\psi$. Since the integral involved is at worst an elliptic integral of the first kind, the relation can be inverted to give the solution $\psi(s)$ explicitly.

In the mass-spring system the constant of integration $h$ equals twice the total energy of the system; but in terms of the wave problem it represents a difference in energies, for $\left(c^{2}-1\right) \psi^{\prime 2}=u_{t}^{2}-u_{x}^{2}$. The second constant of integration represents a translation of the curve $\psi(s)$ along the $s$-axis. Therefore solutions given below are always determined to within an arbitrary phase shift.

It is thus possible to enumerate, in a straightforward way, the various types of solutions of (5), for each combination of values of $\bar{\alpha}$ and $\bar{\beta}$. Rather than carry out a complete classification, however, we shall discuss several important cases which illustrate the interesting wave properties of (1).

3. Continuous range of propagation speeds. Restriction on wave shape. Suppose $\alpha>0, \beta>0$ and $c^{2}>1$, so that $\bar{\alpha}$ and $\bar{\beta}$ are both positive. Equation (5) is now the equation of a mass-spring system with a "hard" restoring force. In (7) $h$ must be positive, and all motions are oscillatory. As shown in [4, p. $20 \mathrm{ff}.], \psi(s)$ is then a cosine type of Jacobian elliptic function: ${ }^{2}$

$$
\psi(s)=a \mathrm{cn}\left[\left(\bar{\alpha}+\bar{\beta} a^{2}\right)^{1 / 2} s\right],
$$

periodic in $s$ with period

$$
L=4(2)^{1 / 2} \int_{0}^{\pi / 2}\left(2 \bar{\alpha}+\bar{\beta} a^{2}+\bar{\beta} a^{2} \sin ^{2} \theta\right)^{-1 / 2} d \theta .
$$

For fixed $\bar{\alpha}$ and $\bar{\beta}$, the amplitude $a$ is determined by $h$; it is given by

2 Waves having the form of the square of the cn function are encountered in the theory of water waves (e.g., see [2]); they are called "cnoidal waves." 


$$
a^{2}=\frac{-\bar{\alpha}+(\bar{\alpha}+2 \bar{\beta} h)^{1 / 2}}{\bar{\beta}} .
$$

By the use of equations (6), (9) is rewritten

$$
L=4(2)^{1 / 2}\left(c^{2}-1\right)^{1 / 2} \int_{0}^{\pi / 2}\left(2 \alpha+\beta a^{2}+\beta a^{2} \sin ^{2} \theta\right)^{-1 / 2} d \theta
$$

Since $s=x-c t$ is essentially a space variable, $L$ denotes the wavelength of a periodic wave in the form of the elliptic function (8), with amplitude $a$ and velocity $c$. For fixed $x, \psi(x-c t)$ is periodic in $t$ with period

$$
P=L / c .
$$

These waves are all supercritical; there are no subcritical waves in the case $\alpha>0, \beta>0$. For if $c^{2}-1<0, \bar{\alpha}$ and $\bar{\beta}$ are both negative, and then (5) and (7) possess no solutions which remain bounded for all $s$ (except the trivial one $\psi(s) \equiv 0$ ).

Thus, for fixed $\alpha>0$ and $\beta>0$, the only wave solutions of (1) are of the form

$$
u(x, t)=\psi(x-c t)=a \operatorname{cn}\left[\left(\frac{\alpha+\beta a^{2}}{c^{2}-1}\right)^{1 / 2}(x-c t)\right]
$$

for arbitrary values of $a$ and $c$, provided $c^{2}>1$.

Note that for fixed $c$, both the wavelength $L$ and period $P$ decrease with increasing $a$. For fixed $a, L$ is proportional to $\left(c^{2}-1\right)^{1 / 2}$ while $P$ is proportional to $\left(c^{2}-1\right)^{1 / 2} / c$. For very large $|c|$, then, $P$ becomes independent of $c$, depending on $\alpha, \beta$ and $a$ exactly as does the period of oscillation of a nonlinear mass-spring system with spring force $-\alpha \psi-\beta \psi^{3}$.

4. Duality between subcritical and supercritical waves. We have seen that if $\alpha$ and $\beta$ are positive, there exist only supercritical wave solutions. But the existence of waves bounded for all $x$ and $t$ depends only on the signs of $\bar{\alpha}$ and $\bar{\beta}$ in (5), and these remain positive if $\alpha, \beta$ and $c^{2}-1$ are all taken negative. On the basis of the preceding discussion, we conclude that if $\alpha<0$ and $\beta<0$, there can be only subcritical waves; again, these are periodic waves, with arbitrarily prescribed (subcritical) velocity $c$ and amplitude $a$. Indeed, equations (10-13) carry over almost without change.

More generally, since changing the signs of $\alpha, \beta$ and $c^{2}-1$ leaves $\bar{\alpha}$ and $\bar{\beta}$-and therefore the wave profiles-unchanged, we have the following duality between sub- and supercritical waves: 
Suppose that when $\alpha$ and $\beta$ have the fixed values $\alpha_{0}$ and $\beta_{0}$ respectively, $u(x, t)=\psi_{0}\left(x-c_{0} t\right)$ is a wave solution of $(1)$, where $0<c_{0}^{2}<2$. Then there is $a$ wave $u(x, t)=\psi_{0}\left(x-c^{\prime} t\right)$ for $\alpha=-\alpha_{0}, \beta=-\beta_{0}$ and $c^{\prime 2}-1=1-c_{0}^{2}$. This is true for periodic and nonperiodic waves.

It is not hard to understand on physical grounds why the waves must propagate at speeds greater (less) than unity when $\alpha$ and $\beta$ are positive (negative), i.e., when the terms on the right of (1) represent a distributed attractive (repulsive) force. All wave solutions of (2) travel at unit speed. Also the wave speed in a string increases with the tension, and since a distributed restoring (repulsive) force effectively increases (decreases) the tension, we get propagation velocities greater (less) than unity.

5. Periodic and nonperiodic waves. In the cases mentioned so far, where $\alpha \beta>0$, only periodic waves exist. If $\alpha \beta<0$, however, there are periodic and nonperiodic waves.

Suppose, for example, that $\alpha>0, \beta<0$, and $c^{2}>1$, so that $\bar{\alpha}>0$, $\bar{\beta}<0$; this is the case of a "soft" spring, which exerts an attractive force for small $|\psi|$ and a repulsive force for large $|\psi|$. It is not hard to see that, if $0<h<-\bar{\alpha}^{2} / 2 \bar{\beta}$, (7) has periodic solutions $\psi(s)$ of the form (8), with period $L$ and amplitude $a$ given by (9) and (10) respectively. ${ }^{3}$ In contrast with the case $\bar{\beta}>0$, now $L$ increases with increasing $a$.

If $h<0$ or $h>-\bar{\alpha}^{2} / 2 \bar{\beta}$, the solutions are unbounded.

If we set $h=-\bar{\alpha}^{2} / 2 \bar{\beta}$ in (7), we obtain

$$
\psi(s)= \pm(-\bar{\alpha} / \bar{\beta})^{1 / 2} \tanh \left[(\bar{\alpha} / 2)^{1 / 2} s\right] .
$$

In other words, for given $\alpha>0$ and $\beta<0$, there are supercritical periodic and nonperiodic waves. The periodic ones are of the form (13), with any velocity $c$ and amplitude $a$ satisfying $c^{2}>1$ and $0<a<(-\alpha / \beta)^{1 / 2}$. The nonperiodic waves are of the form

$$
\begin{aligned}
& u(x, t)=\psi(x-c t) \\
&= \pm\left(\frac{-\alpha}{\beta}\right)^{1 / 2} \tanh \left[\left(\frac{\alpha}{2\left(c^{2}-1\right)}\right)^{1 / 2}(x-c t)\right]
\end{aligned}
$$

they all have the amplitude $(-\alpha / \beta)^{1 / 2}$ and the same general shape, but the steepness of each wave depends upon its velocity $c$. A nonperiodic

${ }^{3}$ In this case there are also unbounded solutions. Indeed, for each value of $h$ in the range $0<h<-\bar{\alpha}^{2} / 2 \bar{\beta}$, the graph of (7) in a $\psi, \psi^{\prime}$-plane (the so-called "phase trajectory" of the solution) consists of 3 disconnected branches: one is a closed curve, representing the periodic solution, while the other two are open, unbounded curves. 
solution of the form (14) is unstable, in the sense that small deviations from it produce either periodic or unbounded solutions.

While there are no continuous wave solutions of (1) with velocities $c= \pm 1$, we see that discontinuous waves are obtained by letting $c$ approach +1 or -1 . In the limit the solution (14) takes the form of a step function traveling at unit speed. (This is not possible in the cases $\alpha \beta>0$.) An observation of this sort is made in [3], where the waves have a form similar to (14).

Two further remarks are in order. First of all, in the present case $(\alpha>0, \beta<0)$ there exist also subcritical waves, in contrast with the cases $\alpha \beta>0$, where for given $\alpha$ and $\beta$ we can not have both sub- and supercritical waves. This is readily seen from the fact that $\alpha>0$, $\beta<0, c^{2}-1<0$ yield $\bar{\alpha}<0, \bar{\beta}>0$, in which case the spring force $-\bar{\alpha} \psi-\bar{\beta} \psi^{3}$ in (5) is repulsive for small values of $|\psi|$ and attractive for large $|\psi|$. On physical grounds we can conclude that all the motions of such a mass-spring system (and therefore all solutions of (5)) are bounded. Most of these solutions are periodic, some are nonperiodic, but all yield wave solutions of (1). It turns out that the subcritical waves for $\alpha>0, \beta<0$ are more varied in nature than the supercritical ones.

Finally, making use of the duality principle, we see that for velocities in the range $0<c^{2}<2$ the subcritical (supercritical) waves in the case $\alpha<0, \beta>0$ have the same properties as the supercritical (subcritical) waves in the case $\alpha>0, \beta<0$.

\section{REFERENCES}

1. F. A. Ficken and B. A. Fleishman, Initial value problems and time-periodic solutions for a nonlinear wave equation, Comm. Pure Appl. Math. vol. 10 (1957) pp. 331-356.

2. J. B. Keller, The solitary wave and periodic waves in shallow water, Comm. Pure Appl. Math. vol. 1 (1948) pp. 323-339.

3. Balth. van der Pol, On a generalization of the nonlinear differential equation, $d^{2} u / d t^{2}-\epsilon\left(1-u^{2}\right) d u / d t+u=0$, Nederl. Akad. Wetensch. Indag. Math. vol. 19 (1957) pp. $477-480$.

4. J. J. Stoker, Nonlinear vibrations, Interscience, 1950.

Rensselaer Polytechnic Institute 